\title{
The editorial on the special issue
}

\section{'Are sequential sampling models the future gold standard of cognitive psychology?'}

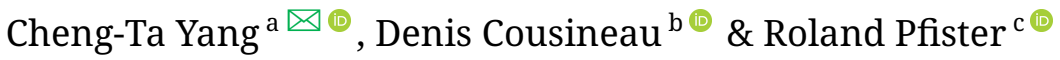 \\ ${ }^{a}$ National Cheng Kung University \\ ${ }^{\mathrm{b}}$ Université d'Ottawa \\ ${ }^{\mathrm{c}}$ Universität Würzburg
}

Abstract $\square$ This editorial introduces the special issue dedicated to sampling models.

Keywords - Sequential sampling models, Decision making, Response times.

yangct1115@gmail.com

$\begin{array}{lr}\text { Acting Editor } & \\ \text { Cheng-Ta } & \text { Yang } \\ \text { (Department } & \text { of } \\ \text { Psychology, } & \text { Na- } \\ \text { tional Cheng } & \text { Kung } \\ \text { University) } & \end{array}$

10.20982/tqmp.16.2.p071

\section{Introduction}

Sequential sampling models assume that people make decisions by gradually accumulating noisy information until reaching a decision threshold. Specifically, evidence is accumulated at a certain rate for a given person working on a given task - known as the drift rate - until the level of evidence meets the decision threshold for a particular response option. Sequential sampling models well explain the relationship between accuracy and response time (RT) in choice RT tasks, and have been elaborated especially for two-alternative forced choice (2AFC) tasks.

Sequential sampling models comprise several families of models (Ratcliff \& Smith, 2004), which differ regarding (1) how the evidence is sampled (i.e., discrete with equallyspaced time intervals, discrete with randomly-spaced time intervals, or continuous), (2) how the evidence is accrued (i.e., fixed- or variable-sized chunks), and (3) the decisional stopping rule (i.e., absolute stopping rule or relative stopping rule). These families include random walk models, diffusion models, accumulator models, and Passion counter models, among others. All types of models have been widely applied and seen rapid development in the last decade.

The current special issue feature aims at providing a broad coverage of these models, including methodological introductory papers, historical perspectives (including an outlook on desirable future developments), recent advances, and hands-on tutorials. In addition, this spe- cial issue links the sequential sampling models with other advanced theory and methodology (e.g., systems factorial technology) to study human decision-making. In detail, the contributions to the special issue are as follows.

Evans and Wagenmakers (2020) give a brief overview about the evidence accumulator model (EAM). Although EAM has been widely used to study the speeded decisionmaking behavior, there are limitations in the implementation of EAM. This article offers several recommendations for researchers to help overcome these limitations, aiming to promote EAM as a standard analysis tool to study the cognitive processes in choice behavior, including individual-difference approaches to cognition.

Fitousi (2020) focuses on linear ballistic accumulator (LBA) models and addresses how the parameters of these models are linked to other analyses of RT distributions, specifically via ex-Gaussian modelling. Results of a structured comparison suggest that researchers should be cautious with using the ex-Gaussian parameters to infer the cognitive stages.

Lerche and Voss (2020) demonstrate that relying only on descriptive measures such as accuracy and RT may at times yield incorrect conclusions regarding the underlying cognitive processes. Specifically, their simulation results demonstrate that different sets of diffusion parameters may produce identical patterns of behavioral results. Strong claims about cognitive processing might thus require model-based analyses.

Wieschen, Voss, and Radev (2020) address statistical as- 
sumptions underlying such diffusion modeling. They argue that the common assumption of Gaussian noise might not be the optimal description of decision-making. Instead, Lévy flights, incorporating more heavy-tailed, nonGaussian noise, might provide a more accurate description of actual decision processes. This argument is backed up by a demonstration that the complex Lévy flight model comes with a superior fit to traditional Gaussian noise models.

Two papers collected in the special issue provide useful statistical tools to fit the data with the sequential sampling models. Lin and Strickland (2020) introduce an efficient and practical software written in $\mathrm{C}++$, accompanied by a step-by-step guide to fit the EMA with Bayesian methods. T.-Groulx, Harding, and Cousineau (2020) discuss the EZ diffusion model, and provide computational tools (R, Excel, SPSS, and Mathematica code) to fit the parameters in a same-different task.

Innes and Kuhne (2020) present an intriguing example of how LBA analysis can be used to explore individual differences in cognitive processing. Two groups of participants (students and Royal Australia Air Force) participated in the experiment, tested with a multiple-object tracking task. Results revealed individual differences in accuracy and response time and trace these differences back to divergent decision criteria and information accumulation rates.

The last article by Townsend, Liu, Zhang, and Wenger (2020) links sequential sampling models to systems factorial technology. This is achieved via systematic simulations using the parallel linear dynamic models to test decisionmaking properties such as architecture, stopping rule, and capacity.

We hope that these contributions help to promote the use of sequential sampling models as an elegant analysis tool to decipher the cognitive mechanisms underlying human decision-making behavior.

\section{References}

Evans, N. J., \& Wagenmakers, E.-J. (2020). Evidence accumulation models: Current limitations and future di- rections. The Quantitative Methods for Psychology, 16, 73-90. doi:10.20982/tqmp.16.2.p073

Fitousi, D. (2020). Linking the ex-gaussian parameters to cognitive stages: Insights from the linear ballistic accumulator (LBA) model. The Quantitative Methods for Psychology, 16, 91-106. doi:10.20982/tqmp.16.2.p091

Innes, R. J., \& Kuhne, C. L. (2020). An LBA account of decisions in the multiple object tracking task. The Quantitative Methods for Psychology, 16, 175-191. doi:10. 20982/tqmp.16.2.p175

Lerche, V., \& Voss, A. (2020). When accuracy rates and mean response times lead to false conclusions: A simulation study based on the diffusion model. The Quantitative Methods for Psychology, 16, 107-119. doi:10 . 20982/tqmp.16.2.p107

Lin, Y.-S., \& Strickland, L. (2020). Evidence accumulation models with R: A practical guide to hierarchical bayesian methods. The Quantitative Methods for Psychology, 16, 133-153. doi:10.20982/tqmp.16.2.p133

Ratcliff, R., \& Smith, P. L. (2004). A comparison of sequential sampling models for two-choice reaction time. Psychological review, 111, 333-367. doi:10 . 1037 / 0033 295X.111.2.333

T.-Groulx, J., Harding, B., \& Cousineau, D. (2020). The EZ diffusion model: An overview with derivations, software and an application to the "same"-“different" task. The Quantitative Methods for Psychology, 16, 154-174. doi:10.20982/tqmp.16.2.p154

Townsend, J. T., Liu, Y., Zhang, R., \& Wenger, M. J. (2020). Interactive parallel models: No Virginia, violation of miller's race inequality does not imply coactivation and yes Virginia, context invariance is testable. The Quantitative Methods for Psychology, 16, 192-212. doi:10.20982/tqmp.16.2.p192

Wieschen, E. M., Voss, A., \& Radev, S. (2020). Jumping to conclusion? A lévy flight model of decision making. The Quantitative Methods for Psychology, 16, 120-132. doi:10.20982/tqmp.16.2.p120

\section{Citation}

Yang, C.-T., Cousineau, D., \& Pfister, R. (2020). The editorial on the special issue 'Are sequential sampling models the future gold standard of cognitive psychology?’ The Quantitative Methods for Psychology, 16(2), 71-72. doi:10.20982/tqmp. 16.2.p071

Copyright @ 2020 , Yang, Cousineau, and Pfister. This is an open-access article distributed under the terms of the Creative Commons Attribution License (CC BY). The use, distribution or reproduction in other forums is permitted, provided the original author(s) or licensor are credited and that the original publication in this journal is cited, in accordance with accepted academic practice. No use, distribution or reproduction is permitted which does not comply with these terms.

Received: $11 / 03 / 2020$

The Quantitative Methods for Psychology 